\section{Prävention in der ärztlichen Versorgung - Potenziale, Wirksamkeit und Umsetzung}

\author{
Die Kunst zu heilen kann viele Leiden lindern, \\ doch schöner ist die Kunst, die es versteht, \\ die Krankheit am Entstehen schon zu hindern.
}

Max von Pettenkofer (1818-1901)

Trotz der bisherigen und noch zu erwartenden therapeutischen Fortschritte steht bei den heute dominierenden chronischen Erkrankungen meistens fest, dass eine Wiederherstellung der Gesundheit nicht zu erwarten ist. Krankheitsorientierte Prävention und "salutogen" orientierte Gesundheitsförderung (s. Kasten) werden deshalb als ein wichtiger Ansatz zur Verbesserung der Gesundheit gesehen. Mittel- und langfristiges Ziel ist es, nicht nur die Lebenserwartung zu erhöhen (hier nimmt Deutschland unter den Industrienationen nur eine Mittelstellung ein), sondern vor allem die Qualität der Lebensjahre zu verbessern und altersabhängige Krankheitslasten zu verhindern bzw. hinauszuschieben. Damit könnte gleichzeitig der demographisch bedingte Zusatzversorgungsbedarf bei HerzKreislauf-Erkrankungen, Erkrankungen des Bewegungsapparates, Krebserkrankungen, obstruktiven Lungenerkrankungen sowie Demenz vermindert werden. Die WHO wies bereits in ihrem Weltgesundheitsbericht 1998 darauf hin, dass die Last des demographischen Umbaus nur bewältigt werden kann, wenn mehr in mittel- und langfristige Prävention investiert wird. In Deutschland ließen sich rund 24-30\% der derzeitigen Gesundheitsausgaben langfristig durch Prävention vermeiden.

Die Notwendigkeit und Bedeutung der Prävention und Gesundheitsförderung kommt in zwei aktuellen Sachverständigenberichten der Bundesregierung zum Ausdruck. Das am 20. März diesen Jahres vom Sachverständigenrat für die Konzertierte Aktion im Gesundheitswesen vorgestellte neue Gutachten »Bedarfsgerechtigkeit und Wirtschaftlichkeit» befasst sich im ersten von drei Bänden intensiv mit der Krankheitslast sowie den Potenzialen und der Wirksamkeit von Prävention. Auch der zu Beginn 2001 erschienene 3. nationale Altenbericht der Bundesregierung "Altern und Gesellschaft" setzt sich intensiv mit den Mög- lichkeiten der Prävention und Gesundheitsförderung auseinander.

\section{Politische Zeichen}

Auf politischer Ebene wurden mit der Wiedereinführung der primären Prävention im Rahmen des Gesundheitsreformgesetzes 2000 ( $§ 20$ SGB V) deutliche Zeichen für eine Gesundheitsorientierung gesetzt. Ziel der kassenfinanzierten und ausdrücklich an Bedarf, Zielgruppen, Methoden und Zugangswegen ausgerichteten Prävention soll es sein, den allgemeinen Gesundheitszustand zu verbessern und sozial bedingte Ungleichheit von Gesundheitschancen zu vermindern. Mit dem Gesundheitsreformgesetz wurde gleichzeitig die Eigenverantwortung und Kompetenz der Versicherten gestärkt - durch den $\S 65 \mathrm{~b}$ SGB V zur "Förderung von Einrichtungen zur Verbraucher- und Patientenberatung" und die Einführung von Patientenschulungen für chronisch Kranke und ihrer Angehörigen als ergänzende Leistungen der Rehabilitation ( $§ 43$ SGB V).

Den Ärzten kommt als den wichtigsten Ansprechpartnern für die Gesundheit der Patienten eine bedeutende Rolle in der Prävention und Gesundheitsförderung zu. Mit ihrer Hilfe können Risikopersonen identifiziert, über für sie geeignete Präventionsmaßnahmen informiert und zu einer Teilnahme motiviert werden. Diese Beratungstätigkeit erfordert angemessene Kenntnisse über die Potenziale und Wirksamkeit von Präventionsmaßnahmen für definierte Krankheiten und präventive Einrichtungen.

Für die klinische Prävention liegen in Kanada und den USA zielgruppenorientierte Empfehlungen zur Durchführung präventiver Maßnahmen vor, die sich an evidenzbasierte Standards anlehnen. Von der EU wurde im Jahr 2000 ein Bericht der International Union for He-

\section{Definitionen*}

Primärprävention bedeutet, Teilursachen (Risikofaktoren) von definierten Erkrankungen zu vermeiden oder sie zu erkennen und zu beeinflussen. Sie setzt vor Eintritt einer fassbaren biologischen Schädigung ein. Ziel der Primärprävention ist die Reduktion der Inzidenz oder der Eintrittswahrscheinlichkeit bei einem Individuum bzw. einer (Teil-)Population. Beispiele für primärpräventive Maßnahmen sind Sturzprophylaxe im Alter und Jodprophylaxe.

Sekundärprävention bezieht sich auf die Entdeckung eines eindeutigen (noch symptomlosen) Frühstadiums einer Krankheit, ein frühes Eingreifen nach der Manifestation einer Krankheit und ihre Frühtherapie. Diese Frühtherapie sollte gegenüber einer später einsetzenden Normalbehandlung einen gesicherten Zusatznutzen aufweisen, um unnötiges Leid, unnötige Kosten und Risiken zu vermeiden. Als Sekundärprävention wird in jüngster Zeit auch verstanden, das Wiederauftreten einer Krankheit nach erfolgter Ersterkrankung zu verhindern. Sie sollte immer auch Bestandteil von Behandlungskonzepten sein. Zu den sekundärpräventiven Maßnahmen zählt die Krebsfrüherkennung und verhaltensbezogene Maßnahmen zur Vermeidung eines Reinfarktes nach einem Herzinfarkt. Tertiärprävention ist die Behandlung einer symptomatisch gewordenen Krankheit mit dem Ziel, Rezidive zu verhindern und die Leistungsfähigkeit wiederherzustellen. Damit ergeben sich Überschneidungen mit der Kuration. Konzepte der Tertiärprävention beziehen sich deshalb nur auf Interventionen zur Verhinderung bleibender Funktionseinbußen. Tertiärprävention ist ein wichtiger Teil der Rehabilitation und hat immer auch präventiven Charakter. Beispiele für Tertiärprävention sind verhaltensbezogene Maßnahmen nach einer Krebserkrankung. Gesundheitsförderung setzt nach der Ottawa-Charta der WHO (1986) vor allem bei der Stärkung von GesundheitsRessourcen an, d.h. den individuellen physischen und psychischen Bewältigungsmöglichkeiten von Gesundheitsbelastungen. Außerdem zielt sie auf gesellschaftspolitische Veränderungen mit dem Ziel, Ungleichheiten in der Gesundheit zu vermindern. Zur Gesundheitsförderung zählen neben einer gesunden Ernährung auch Maßnahmen zur Erhöhung von Selbstbewusstsein, Verbesserung der Bildung und gesundheitsbezogene Optimierung von Arbeitsstrukturen. * nach Sachverständigenrat 2000/2001 


\section{Jodprophylaxe*}

Angemessene Häufigkeit der zu verhindernden Gesund heitsstörung:

In Deutschland, das zu den Jodmangelgebieten zählt, zeigen mindestens $40 \%$ der Bevölkerung eine mittels Ultraschall nachweisbare Schilddrüsenvergrößerung.

Medizinische Relevanz: Etwa 90000 Bundesbürger pro Jahr müssen sich einer Schilddrüsenoperation unterziehen und benötigen danach in der Regel eine lebenslange medikamentöse Therapie.

Vollkswirtschaftliche Relevanz:

Die geschätzten Kosten belaufen sich auf jährlich 2 Milliarden DM.

Nachgewiesene Wirkung: Das JodMonitoring von 1996 konnte nachweisen, dass sich durch Präventionsmaßnahmen (Aufklärung, Verwendung von Jodsalz im Haushalt und bei industriell hergestellten Lebensmitteln) die Jodversorgung bei Erwachsenen von 1975-96 von 30-70 $\mu \mathrm{g}$ pro Tag auf $111-126 \mu$ g pro Tag verbessert hat (von der Deutschen Gesellschaft für Ernährung werden $200 \mu \mathrm{g}$ pro Tag empfohlen, von der WHO 150). Die Wirksamkeit ist auch erkennbar an der Abnahme der Neugeborenen-Struma von z. B. $6 \%$ (Ulm, Jena) auf 0,1\% durch Gabe von Jodid-Tabletten an alle Schwangeren.

Angemessenheit der Aufwendungen: Die Kosten für die Jodierung des Speisesalzes sind gering.

Risilko der Präventionsmaßnahme: Es sind keine Risiken (Überdosis, Auslösung allergischer Reaktionen u.ä.) bekannt (Dtsch med Wschr 2000; 125: 671-675). Kommentar: Die tägliche Jodaufnahme der deutschen Bevölkerung beträgt immer noch nur etwa zwei Drittel des empfohlenen Richtwertes, so dass weitere Maßnahmen zur verstärkten Benutzung von Jodsalz notwendig erscheinen. Dies gilt sowohl für Privathaushalte (und hier besonders bei Schwangeren und Stillenden), in denen die freiwillige Benutzung von jodiertem Speisesalz auf einen Bevölkerungsanteil von $90 \%$ angehoben werden sollte, als auch für die industrielle Fertigung von Lebensmitteln (z. B. bei der Brotherstellung oder in der Fleischverarbeitung). Auch sollte auf Empfehlung des Arbeitskreises Jodmangel neben dem in Deutschland zugelassenen Jodat auch Jodid zugelassen werden: Durch die Beseitigung des bestehenden Exporthindernisses für Jodid-haltige Lebensmittel kann die Jodprophylaxe weiter verbreitet werden. Es empfiehlt sich darüber hinaus, das Jod-Monitoring von 1996 zu wiederholen, um zu überprüfen, inwieweit weiterer Handlungsbedarf besteht.

*nach Sachverständigenrat 2000/2001 alth Promotion and Education zur Evidenz der Wirksamkeit von Gesundheitsförderung herausgegeben. Entsprechende Empfehlungen für Deutschland gibt es bislang nicht. Hier will die DMW eine Lücke schließen.

Mit der nun beginnenden "Serie ... Prävention» sollen die Möglichkeiten der Prävention für weit verbreitete Gesundheitsstörungen und chronische Krankheiten im ärztlichen Alltag aufgezeigt und Hilfestellungen für die Umsetzung in die Praxis gegeben werden. In regelmäßiger Folge werden Risiken, Gesundheitsstörungen und Krankheiten aufgegriffen, die dem Hausarzt und Kliniker täglich begegnen können: Adipositas, Rauchen, Bluthochdruck, Rükkenerkrankungen, Allergien, Osteoporose, Diabetes etc. Zudem werden die Rolle und Verbesserung der hausärztlichen Versorgung, die Beratung in der Praxis und die Rolle der Krankenkassen in der Prävention thematisiert. Dabei stehen folgende Fragen im Mittelpunkt:

- Wie lassen sich in der Zukunft die krankheitsbedingte Minderung von Lebensqualität und verlorene Lebensjahre am wirksamsten verringern?

- Wie können (sozial schwache) Risikopersonen erreicht werden?

- Welche äußeren und verhaltensabhängigen Risiken und Belastungen des Patienten können reduziert werden?

- Welche persönlichen Ressourcen des Patienten können gestärkt werden?

- Welche Einrichtungen bieten Informationen und Maßnahmen zur Prävention an?

Der Auswahl und Darstellung der einzelnen Krankheiten bzw. Gesundheitsstörungen liegen die vom Sachverständigenrat aufgestellten Priorisierungskriterien zugrunde, nach denen eine Maßnahme für die gesamte Bevölkerung oder einer Risikogruppe empfohlen werden kann (Sachverständigenrat 1994, 1995):

- Die zu vermeidende Gesundheitsstörung soll in einer auf die Bevölkerungsgruppe(n) und die Fragestellung bezogen angemessenen Häufigkeit vorliegen.

- Sie soll medizinisch relevant sein.

- Sie soll auch volkswirtschaftlich bedeutend sein.

- Die Präventionsmaßnahme der zu vermeidenden Krankheit soll wirksam sein und ihr ohne unvertretbare bzw. unerwünschte Risiken vorbeugen.
- Die Intervention sollte den individuellen und kulturellen Werten entsprechen.

- Die Aufwendungen für die vorgesehene Maßnahme sollen angemessen sein (akzeptable Nutzen-Kosten-Relation).

Der Sachverständigenrat hat auf die Latenz zwischen Anfall der Präventionskosten und Einsparung bei Krankheitskosten hingewiesen, die eine Vorfinanzierung erforderlich machen kann. Die rein ökonomische Betrachtung sollte heute Bestandteil eines jeden Vorschlages für eine Präventionsmaßnahme sein. Hierbei müssen nicht nur die Kosten, z. B. der gesetzlichen Krankenversicherung, sondern auch Eigenleistungen und evtl. Arbeitsunfähigkeit berechnet werden, ebenso wie die Erträge, die sich aus eingesparten Behandlungskosten und Produktivitätszuwachs berechnen. Ein Beispiel für die Anwendung der Priorisierungskriterien zeigt der Kasten "Jodprophylaxe».

Wir erhoffen uns von dieser Artikelserie Hinweise auf eine Optimierung der Umsetzung im Sinne von Qualitätsmanagement, Vorschläge für die Finanzierung, Klärung der Zuständigkeit und Koordination von Aktivitäten. Die Festlegung von Zielen für Gesundheitsförderung und Prävention wird erst durch eine aktive Beteiligung der Bevölkerung bzw. von Zielgruppen erfolgversprechend. Aufklärung der Nutzer und die Mitwirkung aller Beteiligten im Gesundheitswesen sind unverzichtbar.

Prof. Dr. Dr. h. c. Peter C. Scriba, München Prof. Dr. Friedrich W. Schwartz, Hannover Dr. Ulla Walter, Hannover

In der nächsten Folge lesen Sie: Adipositas

\section{Fachliche Betreuung der "Serie ... Prävention॥:}

Prof. Dr. Dr. h. c. Peter C. Scriba

Medizinische Klinik / Klinikum Innenstadt der Universität München

Ziemssenstr. 1

80336 München

Prof. Dr. Friedrich W. Schwartz Medizinische Hochschule Hannover Abteilung für Epidemiologie, Sozialmedizin und Gesundheitsforschung Carl-Neuberg-Str. 1 30623 Hannover 